\title{
Data Analysis of Consumption Upgrading of Urban Residents in China
}

\author{
Xiaoyu Wang \\ Nanjing Normal University, Nanjing, China \\ Email: 1842993492@qq.com
}

How to cite this paper: Wang, X.Y. (2019) Data Analysis of Consumption Upgrading of Urban Residents in China. Open Journal of Social Sciences, 7, 15-31.

https://doi.org/10.4236/jss.2019.710002

Received: September 10, 2019

Accepted: October 6, 2019

Published: October 9, 2019

Copyright $\odot 2019$ by author(s) and Scientific Research Publishing Inc. This work is licensed under the Creative Commons Attribution International License (CC BY 4.0).

http://creativecommons.org/licenses/by/4.0/

\begin{abstract}
In the process of deepening the supply-side reform, China's economic operation has shown a good momentum of steady development, and consumption has begun to play a prominent role in promoting economic growth. However, there are still many different opinions on whether it is "consumption upgrade" or "consumption downgrade". Based on the eight types of consumption data of urban residents in China from 1993 to 2016, this paper uses the time-series global principal component analysis method and the ELES model to empirically analyze the longitudinal time series data from the three levels of consumption level, consumption structure and consumer satisfaction. The results show that the consumption level of urban residents is increasing steadily, the consumption structure is continuously optimized, and consumer satisfaction is significantly improved. At this stage, China is indeed in a period of consumption upgrading, rather than the consumption reduction proposed by the private sector. Therefore, under the background of supply-side structural reform, measures such as improving the income distribution system, improving the quality of labor supply, and developing scientific and medical equipment are the fundamental ways to accelerate the upgrading of China's consumption.
\end{abstract}

\section{Keywords}

Consumption Upgrade, Time-Series Global Principal Component Analysis, ELES Model, Supply Side Reform

\section{Introduction}

Since 2015, China's economy has entered a new normal, showing that although consumption has risen, the economic growth rate is slow, which is caused by the "mismatch of supply and demand". Mao Zhonggen (2019) pointed out that the 
main problems faced by China's consumption development at this stage are the poor quality of the consumer environment, the imperfect consumption system, the lack of supply of high-end consumer goods and services, and the inferiority of products and consumption inequality [1] [2]. The central government proposed a supply-side structural reform. Premier Li Keqiang (2019) proposed that in the past year, the supply-side structural reforms will be deepened, the vitality of the real economy will be continuously released, and consumption will continue to escalate [3] [4]. On the other hand, according to Maslow's hierarchy of needs, as people's living standards improve, people's needs will develop from lower levels of meeting physiological security needs to higher levels of respect and self-realization, manifested as consumption upgrades. This paper combines the previous research literature to define consumption upgrading as follows: the process of continuous improvement of consumption level, optimization of consumption structure, and transformation of consumption quality from low level to high level. At the same time, the frequency and variety of consumers are gradually expanding, and the consumption concept is more open and innovative. People gradually pay attention to the quality and mode of consumption, and the consumption style shifts from the low end to the high end, also the consumption content is from material to spiritual.

Li Pumin (2019) believes that today's consumption presents a series of prominent changes, from quantity to quality, from imitative consumption to personalized and diversified consumption [5]. The overall scale of household consumption continues to expand, the consumption power of residents continues to increase, the consumption content is more diversified, the consumption pattern is more convenient, and consumers pay more attention to their own value and sustainable development. But it still exists in the process of consumption upgrade the latter problem such as insufficient consumer supply, residents' ratio rise too fast; these phenomena have caused some residents to feel the decline in the consumption experience, such as Shi Xinran (2019), the contribution rate of GDP and consumption to GDP. As well as data on per capita disposable income, the study found that survival-type consumption still dominates [6]. Therefore, "consumption downgrade" has become a hot word since 2018. Many people cite a lot of cheap shopping platforms to prove that young people's propensity to consume tends to downgrade. This paper will explain that China's urban residents are in the consumption upgrading stage from three aspects: consumption level, consumption structure and consumer satisfaction. In the past, the research on consumption upgrading only involved two aspects of consumption level and consumption structure. This paper proposes the level of consumer satisfaction and analyzes it through the model. The time-series global principal component analysis method is used to measure the consumption structure optimization degree based on the eight major consumption data of urban residents in China from 1993 to 2016. The comprehensive score is given and the time-series curve is drawn. Then the ELES model is used to measure the marginal tendency of 8 
categories of consumption items to study consumer satisfaction.

\section{Literature Review}

Consumption downgrade is relative to consumption upgrade. Consumption degradation generally shows an increase in the proportion of consumers' survival consumption, or a certain reduction in efficiency, experience, personality, taste and attitude, or consumers are low-quality, the pursuit of low-cost and inferior products. At present, some domestic scholars have given corresponding theoretical explanations for the argument that "China is now in the stage of consumption upgrading or consumption degradation". Ma Zhengui (2018) believes that the consumers who are keen on shopping on Pin Duo Duo may not have enough spending power, but if the consumption level of the third or fourth tier continuously stimulates an emerging consumption life, it is also their "consumption upgrading" [7]. Ma Qiji (2018) also believes that the "marginal business" and "marginal economy" methods have been adopted in shopping platforms such as Pin Duo Duo, and the low-quality and low-priced products, along with the counterfeit and inferior products constitute a "low-end market" to meet a part. The demand, this part of consumers in the pursuit of high cost performance is not in the consumer downgrade [8]. Xue Junmin (2019) analyzed that the diversification of food consumption led to the diversification of consumers' choices, and the upgrading of food products, which inhibited the reduction of Engel's coefficient. Therefore, this phenomenon is not a demotion of consumption, but a upgrading of high-quality consumption [9]. Huang Juan (2019) found that the high-level consumption expenditure of Chinese residents in 2016 increased by nearly 3 percentage points compared with 2013 [10]. At the same time, there is also a voice supporting the theory of consumer downgrade. Li Meng (2018) based on the latest statistics, In the first half of the year2018, the growth rate of China's total retail sales of consumer goods reached its lowest level in 15 years. In July, the sales growth of domestic large-scale retail enterprises decreased by $3.9 \%$. He believes that China is currently in a period of consumption downgrade [11]. Kuang Xianming (2018) refuted this. He believes that consumption upgrading should be based on the continuous increase in the proportion of service consumption in the consumption structure of urban and rural residents. The growth rate of total retail sales of consumer goods cannot objectively measure the trend of consumption development [12]. In terms of judging whether consumption is degrading or not, Tan Haojun (2018) believes that the key depends on whether the consumption pattern shifts from the middle to the high end, whether the consumption content shifts from the spirit to the substance, and whether the consumer's concern shifts from quality to quantity [13]. Sun Feng (2019) used the theoretical research of consumer sociology to find that functional consumer demand tends to be degraded, meaning consumer demand tends to escalate, and symbolic consumption plays an increasingly important role in consumer taste and fashion [14]. Shi Mingming, Jiang Zhou and Zhou Xiaoyan 
(2019) analyzed the optimization process of China's consumption structure, and found that residents' consumption showed the characteristics from survival consumption to pleasure consumption and development consumption, and from material consumption to service consumption, namely consumption upgrading. Although the level of household consumption and consumption quality have generally escalated, some groups of people have degraded consumption phenomena. This article also provides an explanation for the phenomenon that "low-quality and inferior product sales are rising". On the one hand, the growth of housing expenditure in recent years indicates that other consumer expenditures have been squeezed out. On the other hand, people with higher Engel coefficient are sensitive to current income or have the characteristics of large inter-period consumption fluctuation due to budget constraints and consumption habits, which inhibits the improvement of consumption intention [15]. Sun Hao (2019) studied China's consumption upgrade in terms of income growth, technological progress, and rising housing prices from the perspective of "consumption upgrading" and "consumption stratification". He believes that technological progress and income growth together promote household consumption upgrades. Exogenous shocks such as rising housing prices will have different effects on budget constraints of different groups, leading to consumption differentiation and consumption stratification, so consumption degradation is an illusion [16]. Sun Xingjie et al. (2019) used the inter-provincial panel data from 2002 to 2017 to analyze the trend of China's household consumption changes from three levels: urban and rural, regional and income levels. China's consumer market shows the characteristics of consumption stratification, which is not the difference between "consumption upgrading" and "consumption demotion". In other words, the change of residents' consumption in the short term is mainly the difference of consumption upgrading speed between different groups [17].

Contributions of this paper: First of all, most scholars have studied from the perspective of the current situation and influencing factors, and these studies are based on their recognition of China's current stage of consumption upgrading. For the debate between the official and private consumption upgrades and consumer downgrades, although a small number of articles give their own understanding through textual combing, there is currently no literature to fully demonstrate from the perspective of data and models. Secondly, this paper puts forward an analysis of the level of consumer satisfaction, which is more innovatively measured by the indicator of marginal propensity to consume.

\section{Theoretical Analysis}

Scholars have studied the issue of consumption upgrading or downgrading from various angles. For the existence of consumption degradation, this section will from the level of consumption, consumer satisfaction and the consumption structure of the three level, combined with the national bureau of statistics released in 1993-2016 annual data and theory analysis to our country urban resi- 
dents consumption upgrading.

\section{1) Level of consumption level}

Consumption level is an indicator to measure the satisfaction degree of social members' own utility and the improvement degree of material and cultural residence standards after they purchase and use social products or services through personal income consumption. The level of consumption can be set from the per capita consumption expenditure and the total retail sales of social consumer goods. The total retail sales of social consumer goods reflect the development of the people's material and cultural residence standards and the realization of the purchasing power of social goods during a certain period of time. The per capita consumption expenditure here consists of eight parts: food, clothing, housing, household equipment and services, health care, transportation and communications, education, culture and entertainment, and other commodities.

According to the data released by the National Bureau of Statistics, from 1993 to 2016, the absolute amount of per capita consumption expenditure of urban residents in China increased with the increase of per capita disposable income, and the growth rate continued to increase, with 2011 to 2016, urban residents spending growth doubled, the consumption level of growth rate of $10 \%$ for five years. It can be divided into two stages: the first stage is 1993-2000, the growth rate of urban residents' consumption level is slow; the second stage was from 2001 to 2016, when the growth rate of residents' consumption level accelerated. The possible reason is that according to the S-S theorem, after China's accession to the WTO, international trade increased the income of a large number of cheap labors in China, which led to an increase in the overall income level of urban residents in China, and the increase in income expanded domestic demand and consumption scale. In addition, from the perspective of the total retail sales of consumer goods, its absolute value has also increased year by year, from 830.01 billion yuan to 332.316 billion yuan. In summary, the absolute annual consumption expenditure of urban residents in China and the total retail sales of social consumer goods have steadily increased year by year, and the consumption level of urban residents in China is also increasing year by year.

\section{2) Level of Consumption structure}

The consumption structure is the proportional relationship of various types of consumption data purchased by people during the consumption process. Among them, the proportion of food expenditure in residents' consumption expenditure is an important indicator to measure the consumption structure of residents, namely Engel coefficient. According to the data from 1993 to 2016, the Engel coefficient of urban residents in China has continued to decline, from $50 \%$ in 1994 to $29.3 \%$ in 2016. This is also in line with Engel's rule of consumption structure change: with the increase of residents' income, the share of expenditure on food will decline. According to the definition of the UN Food and Agriculture Organization, the consumption expenditure of urban residents in China has already crossed the "well-off" from "satisfaction" and entered the stage of "rich". 
At this stage, the residence standards of urban residents in China are improving year by year. Consumers tend to use more consumption expenditures for the pursuit of high-quality consumer goods and service consumption.

Wen Qixiang and Ran Jingfei (2005) believe that the optimization of consumption structure is the process of transforming mainstream consumer demand from low to high level, which can be expressed in two forms. One is that original commodity projects will still be consumed, but the original consumer projects will develop to a higher level. Consumers have higher requirements on basic and traditional consumption, and pay more attention to the quality of food, clothing and other consumer goods. The other is that new and higher-level consumption items have been added, the composition and proportion of consumption have changed, and the consumption structure has become more and more advanced. After consumers meet their basic consumer needs and traditional consumer demand, they will increase more emerging consumption and potential consumption [18]. The data released by the National Bureau of Statistics confirms this view: from 1993 to 2016, food consumption expenditures have always been the largest proportion of urban residents' consumption expenditures, and have been on a downward trend. Cultural and educational entertainment consumer spending, consumer spending and transport and communications during a significant increase the proportion of consumer spending trends, the fastest rise in the proportion of consumer spending, up from 6.6\% in 1993 to $22.2 \%$ in 2016, traffic and communication from 3.8\% in 1993 to increase the proportion of consumer spending in 2016 to $13.8 \%$, and the cultural and educational entertainment consumer spending rose from $9.2 \%$ in 1993 to $11.4 \%$ in 2016. Although the medical consumption expenditure has a rising trend, the change range is small, while the proportion of the clothing consumption expenditure, the proportion of the household equipment and supplies consumption expenditure and the proportion of other consumption expenditure have a small decline trend during the period (Figure 1).

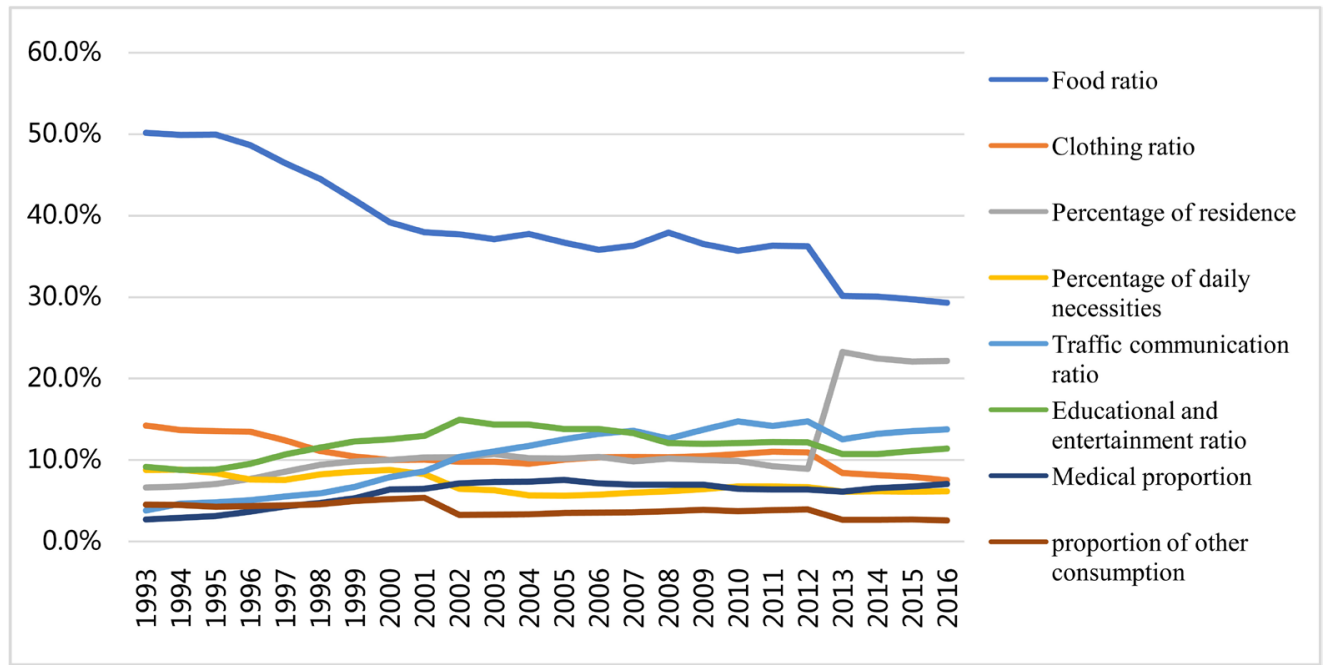

Figure 1. Consumption structure of urban residents in China. 


\section{3) Consumer satisfaction level}

The definition of consumer satisfaction is divided into two categories according to different focuses, one is the expected cost return of the consumer; the other is the consumer's emotional feedback on the consumer experience. The former focuses on the consumer's expected demand for goods or services, while the latter pays more attention to the perceived effect of consumer consumption, namely consumption utility. In this paper, consumer satisfaction is measured by marginal propensity to consume. The higher the utility of a consumer for a certain type of product, the greater the consumer's desire to buy it after the increase in income, and the greater the marginal tendency of a consumer product.

This paper uses the 1993-2016 China Statistical Yearbooks, the per capita disposable income of urban residents and the eight categories of consumer expenditure data divided by the National Bureau of Statistics. Taking disposable income as an independent variable and eight expenditure items as a dependent variable, Eviews 8.0 is used to estimate the ELES model parameters. According to the "Basic Principles of Several Opinions on Improving the Mechanism of Promoting Consumption and Further Encouraging the Consumption Potential of Residents" issued by the Central Committee of the Communist Party of China and the State Council, it is proposed: "with the trend of household consumption upgrading, efforts should be made to increase the supply of high-quality products and services, and to meet basic consumption. Continue to enhance traditional consumption, vigorously cultivate new consumption, and continuously stimulate potential consumption.... Enhance consumers' sense of acquisition, happiness, and security", the eight categories of data of the statistical bureau are specifically subdivided into basic consumption, traditional consumption, emerging consumption and potential consumption. Basic consumption includes food consumption and clothing consumption, while traditional consumption including household goods consumption and other consumption, emerging consumption including transportation and communication consumption, education and cultural consumption and residential consumption, potential consumption, namely, medical and health consumption, using the ELES model to estimate the marginal propensity to consume.

\section{Empirical Analysis}

Based on the urban residents' consumption data from 1993 to 2016, this section uses the time-series global principal component analysis and the ELES model to empirically test the consumption structure and consumer satisfaction, and confirm that there is a consumption upgrade in China.

\section{1) Level of Consumption structure}

The time-series global principal component analysis method is a combination of the classical principal component analysis method and the time-series analysis method. It is based on the classical principal component analysis method, replacing the original global variables with a comprehensive variable, and describes 
the change trajectory of the overall level of the system with time. After introducing the time-series analysis method on the original model of principal component analysis, the expression of the integrated variable is:

$$
Z F=\sum_{i=1}^{k} r_{i} F_{i}
$$

where $r_{i}=\lambda_{i} / \sum_{j=1}^{k} \lambda_{j}, \lambda_{i}$ is the eigenvalue of the correlation coefficient matrix [19].

In this paper, spss18.0 software and time-series global principal component analysis method are used to dynamically describe the consumption structure optimization degree of cities and towns in China from 1993 to 2016. Based on this, the change trajectory of the overall level of the system with time is depicted, and the comprehensive evaluation of the consumption structure optimization degree is predicted. The standardized data of various indicators in the consumption structure of China from 1993 to 2016 are processed. $X_{1}, X_{2}, X_{3}, X_{4}, X_{5}, X_{6}, X_{7}$ and $X_{8}$ respectively correspond to the eight indexes of urban residents' consumption structure data from 1993 to 2016, namely, food, clothing, residence, articles for daily use, transportation and communication, education and culture, medical care and other categories. Correlation analysis of data is required before the time-series global principal component analysis. In Table 1, except for the remaining 7 items of housing category and food, clothing and other categories, the correlation coefficient is relatively low, about 0.8 , and all the other categories are above 0.9.

The normalized data is subjected to time-series global principal component analysis to obtain the principal component eigenvalues and contribution rates of the respective factors. It can be seen from Table 2 that the cumulative contribution rate of the first common factor has reached $95.041 \%$, indicating that the numerical change of the first principal component factor can basically represent the change of the original variable of the indicator system. After determining the principal component, a new variable $\mathrm{F}$ is used to replace the original eight indicators, and it is used as a comprehensive variable of China's consumption structure optimization.

It can be seen from the load factor diagrams in Table 3, the linear combination

Table 1. Correlation coefficient of each indicator.

\begin{tabular}{ccccccccc}
\hline & $X_{1}$ & $X_{2}$ & $X_{3}$ & $X_{4}$ & $X_{5}$ & $X_{6}$ & $X_{7}$ & $X_{8}$ \\
\hline$X_{1}$ & 1 & 0.992 & 0.848 & 0.985 & 0.993 & 0.984 & 0.978 & 0.974 \\
$X_{2}$ & 0.992 & 1 & 0.789 & 0.966 & 0.98 & 0.966 & 0.95 & 0.983 \\
$X_{3}$ & 0.848 & 0.789 & 1 & 0.9 & 0.873 & 0.867 & 0.898 & 0.744 \\
$X_{4}$ & 0.985 & 0.966 & 0.9 & 1 & 0.984 & 0.972 & 0.973 & 0.956 \\
$X_{5}$ & 0.993 & 0.98 & 0.873 & 0.984 & 1 & 0.993 & 0.99 & 0.958 \\
$X_{6}$ & 0.984 & 0.966 & 0.867 & 0.972 & 0.993 & 1 & 0.995 & 0.949 \\
$X_{7}$ & 0.978 & 0.95 & 0.898 & 0.973 & 0.99 & 0.995 & 1 & 0.932 \\
$X_{8}$ & 0.974 & 0.983 & 0.744 & 0.956 & 0.958 & 0.949 & 0.932 & 1 \\
\hline
\end{tabular}


Table 2. Explains the total variance.

\begin{tabular}{ccccccc}
\hline \multirow{2}{*}{ Component } & \multicolumn{3}{c}{ Initial Eigenvalues } & \multicolumn{2}{c}{ Extraction Sums of Squared Loadings } \\
\cline { 2 - 6 } & Total & \% of Variance & Cumulative \% & Total & \% of Variance & Cumulative \% \\
\hline 1 & 7.603 & 95.041 & 95.041 & 7.603 & 95.041 & 95.041 \\
2 & 0.315 & 3.932 & 98.973 & & & \\
3 & 0.052 & 0.653 & 99.626 & & \\
4 & 0.02 & 0.252 & 99.878 & & \\
5 & 0.004 & 0.054 & 99.932 & & \\
6 & 0.003 & 0.032 & 99.965 & & \\
7 & 0.003 & 0.031 & 99.996 & & \\
8 & 0 & 0.004 & 100 & & \\
\hline
\end{tabular}

Extraction Method: Principal Component Analysis.

Table 3. Load coefficient.

\begin{tabular}{cc}
\hline & Component 1 \\
\hline Food $X_{1}$ & 0.995 \\
Clothing $X_{2}$ & 0.979 \\
Residence $X_{3}$ & 0.885 \\
Articles for daily use $X_{4}$ & 0.992 \\
Transportation and Communication $X_{5}$ & 0.997 \\
Education and culture $X_{6}$ & 0.992 \\
Medical care $X_{7}$ & 0.99 \\
Other categories $X_{8}$ & 0.963 \\
\hline
\end{tabular}

expression of the first principal factor is:

$$
\begin{aligned}
Y= & 0.995 X_{1}+0.979 X_{2}+0.885 X_{3}+0.992 X_{4}+0.997 X_{5} \\
& +0.992 X_{6}+0.990 X_{7}+0.963 X_{8}
\end{aligned}
$$

In addition, the absolute value of the load factor reflects the degree of influence of a certain consumption item on the consumption structure of the resident. The larger the absolute value, the greater the impact on the consumption structure. The load factor of traffic and communication consumption is the largest, followed by food, education and culture, daily necessities, health care, clothing, other categories and housing. From the national bureau of statistics, according to data released during the period of 1993 to 2016, the proportion of consumption expenditure of cultural and educational entertainment and transportation and communication in urban residents in China showed a clear upward trend, and the level of medical expenditures increased slightly, more the pursuit of high quality emphasized consumption of urban residents, which significantly promotes the optimization of China's consumption structure. Food consumption and clothing consumption have a greater impact on the optimiza- 
tion of consumption structure. The proportion of food consumption expenditure and clothing consumption expenditure has been declining. The reduction of urban residents' residence consumption expenditure has also significantly accelerated the optimization of China's consumption structure.

Based on the above results, the comprehensive score of China's consumption structure optimization from 1993 to 2016 is calculated and plotted as a time-series (Figure 2). From 1993 to 2016, China's consumption structure has been in the process of continuous optimization. Before 2006, the consumption structure optimization speed was slower. After 2006, the optimization speed has accelerated. Especially from 2007 to 2016, the speed of optimization of China's consumption structure maintained a momentum of sustained and rapid development. Therefore, it is predicted that from 2017 onwards, the consumption structure of urban residents in China will maintain rapid and optimized development.

\section{2) Consumer satisfaction level}

The basic form of the ELES model is:

$$
W_{i}=P_{i} X_{i}=P_{i} X_{i}^{0}+\beta_{i}\left(Y-\sum_{i=1}^{n} P_{i} X_{i}^{0}\right)
$$

where $i=1,2,3, \cdots, n . W_{i}$ is the expenditure of the consumer goods of item I; $P_{i}$ is the price of item I; $X_{i}$ is the basic consumption demand of the item I consumer product; $P_{i} X_{i}^{0}$ Is the basic consumer spending for the consumer goods of item I. $P_{i} X_{i}$ is the total consumption expenditure of item I; $\beta_{i}$ represents the residual marginal propensity to consume the consumer goods of item I after satisfying the basic consumption demand of various consumer goods; $Y$ is real disposable income, and $Y-\sum_{i=1}^{n} P_{i} X_{i}^{0}$ represents the surplus income after the basic consumption expenditure. Making:

$$
\alpha_{i}=P_{i} X_{i}^{0}-\beta_{i} \sum_{i=1}^{n} P_{i} X_{i}^{0}
$$

Finally, the measurement form that is applicable to this analysis model is:

$$
W_{i}=\alpha_{i}+\beta_{i} Y+\varepsilon_{i}
$$

Among them, $i=1,2,3, \cdots, n$ takes the disposable income as the independent

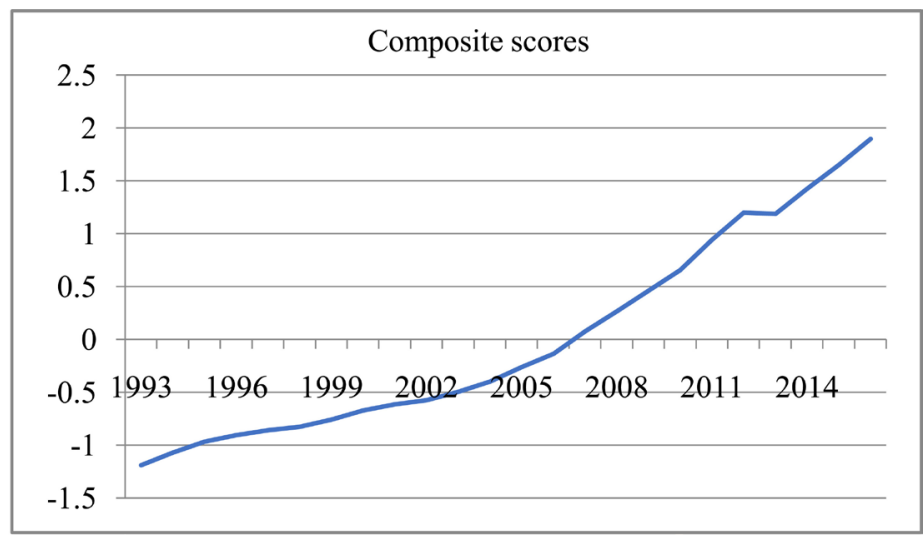

Figure 2. Timing change diagram. 
variable, and the eight major expenditure items as the dependent variables, using Eviews software for linear regression, and the corresponding parameter values are obtained.

From Table 4, it can be seen that all the eight expenditure items have good fitting degree, and the test values of various parameters are also significant. According to the regression results, the marginal propensity to consume among urban residents in China is 0.6567 , which indicates that $65.67 \%$ of the remaining disposable income of urban residents is used to consume these eight categories of consumer goods. According to the table, the marginal propensity to consume of various types of consumption in 1993-2016 is ranked from large to small: food, housing, transportation, culture, education, clothing, medical, daily necessities, and others categories.

According to Table 5, basic consumption includes food consumption and clothing consumption. The marginal consumption tendency of food is higher, reaching $18.3 \%$, while the marginal tendency of clothing is $5.14 \%$. This phenomenon indicates that although the income of urban residents continues to increase, the Engel coefficient of urban residents in China continues to decline, and food is still the main direction and hotspot of household consumption. From the data in the table, even though the basic consumption category is still the main direction of urban residents' consumption, its marginal consumption propensity is still high, but compared with the data from 2008 to 2012, it has

Table 4. Estimation of ELES parameters of urban resident from 1993 to 2016.

\begin{tabular}{ccccccc}
\hline & \multicolumn{1}{c}{$\alpha_{i}$} & $\beta_{i}$ & Std & $R^{2}$ & T value & F value \\
\hline food & 957.5 & 0.183 & 0.0063 & 0.9747 & 29.1106 & 847.43 \\
Clothing & 254.12 & 0.0514 & 0.0029 & 0.931 & 17.2278 & 296.708 \\
Residence & -615.697 & 0.1467 & 0.0134 & 0.8464 & 10.9366 & 119.6089 \\
$\begin{array}{c}\text { Articles for daily use } \\
\text { Transportation and }\end{array}$ & 115.382 & 0.0385 & 0.001 & 0.9853 & 38.3339 & 1469.48 \\
communication & -181.46 & 0.1011 & 0.0026 & 0.9854 & 38.5922 & 1489.36 \\
Education and culture & 169.3334 & 0.0732 & 0.0028 & 0.9692 & 26.3259 & 693.054 \\
Medical care & 0.9751 & 0.0462 & 0.0015 & 0.9764 & 30.2005 & 912.072 \\
Other categories & 112.5901 & 0.0166 & 0.0013 & 0.8832 & 12.9004 & 166.42 \\
total & & 0.6567 & & & &
\end{tabular}

Table 5. Comparison of ELES parameter of urban residents in China in past ten years.

\begin{tabular}{ccccccc}
\hline & \multicolumn{3}{c}{$2008-2012$} & \multicolumn{3}{c}{$2013-2016$} \\
\cline { 2 - 7 } & $\beta_{i}$ & P-value & $R^{2}$ & $\beta_{i}$ & P-value & $R^{2}$ \\
\hline Basic consumption & 0.4611 & 0.0002 & 0.9938 & 0.2998 & 0.0019 & 0.9962 \\
Traditional consumption & 0.1208 & 0.0001 & 0.9977 & 0.087 & 0.0012 & 0.9977 \\
Emerging consumption & 0.3694 & 0.0004 & 0.9911 & 0.5065 & 0.0001 & 0.9979 \\
Potential consumption & 0.0488 & 0.0017 & 0.9748 & 0.1068 & 0.0004 & 0.9991 \\
\hline
\end{tabular}


shown a downward trend in the past five years.

The marginal propensity for traditional consumption, like that of basic consumption, declined between 2008 and 2016. The marginal propensity to consume other categories and daily necessities is $3.85 \%$ and $1.66 \%$, respectively. Consumers' willingness to consume such products is decreasing, but this does not mean that consumer satisfaction with such consumer products is declining. According to the consumption value model proposed by Japanese scholar Kano Kisho, the three categories are distinguished by the degree to which the product and service attributes meet the needs of consumers. Among them, the basic attribute is also called "must have attribute", which refers to the characteristics or attributes that consumers usually think the product and service must have. These attributes of the products do not affect the satisfaction of the consumer, so basic consumption and traditional consumption have no significant impact on consumer satisfaction.

Emerging consumption includes consumption of transportation and communication, education and culture, and residential consumption. The proportion of these three types of consumption has been a large proportion of urban residents' consumption and shows an increasing trend, indicating that urban residents pay more attention to the improvement of material quality of life, the pursuit of spiritual life, and the cultivation of personal quality. The marginal propensity to consume for Residence is $14.67 \%$, ranking second. This indicates that after the income of residents increases, the first consideration for urban residents after securing basic consumption is to increase residential consumption expenditure. With the deepening of the housing system reform and the increase in residents' income, consumers tend to satisfy their pursuit of high-quality life by improving their living environment. Therefore, despite the high housing prices, consumers' willingness to purchase will remain unchanged. The real estate market in some economically developed regions of China has already experienced a shortage of supply. This phenomenon has led to the expansion of consumer housing demand, which has led to an increase in the consumption propensity of urban residents in China. The marginal propensity to consume transportation and communications is $10.11 \%$, ranking third. After satisfying the demand for clothing, food and housing, consumers will put forward higher requirements for "walking". With the popularization of high-tech products such as automobiles and smart phones, transportation and communication products have become new consumer hotspots. Finally, the marginal propensity to consume education, culture and entertainment services is $7.32 \%$, ranking fourth. In recent years, as material needs have been better met, urban residents have become more and more focused on spiritual needs. People have begun to pay attention to the improvement of their cultural quality and the development of recreational activities. Residents are willing to invest large amounts of money in education and entertainment. According to the consumer value model, emerging consumer products have more attractive attributes, also called "surprise attributes," pointing 
out the innovative product and service attributes that consumers expect. When products provide consumers with such attributes and meet the needs of consumers, consumers will be surprised. The rising trend of the marginal consumption propensity of emerging consumption fully demonstrates that consumers' willingness to consume such products is enhanced, that is, their consumption utility increases, or consumer satisfaction increases.

Among several types of consumer goods, in addition to other categories and daily necessities, the potential consumption of consumer products is the lowest, accounting for only $4.62 \%$. This may be because on the one hand, China's urban residents as a whole pay more attention to the amount of food consumed. The problem has only begun to care about health care in recent years, which is related to the fact that China's medical care has been in an imperfect state. On the other hand, the country has paid more and more attention to the reform of the medical system in recent years, and is committed to improving public welfare. Residents enjoy a large number of preferential policies and subsidies in medical treatment. From this point of view, although urban residents in China have less consumption expenditures in health care, they only bear a small part of such consumption expenditures. However, as can be seen from Table 5, its marginal propensity to consume also shows an upward trend, rising from $4.88 \%$ to $10.68 \%$, which means the consumption of medical care has increased. Such consumer goods have a single-dimensional attribute, also referred to as "the more the better" attribute, which refers to the product and service attributes that the consumer desires and expressly requires. The more such attributes a product has, the higher the satisfaction of the consumer will be. Due to the improvement of living standards and the improvement of the concept of life in recent years, people have paid more attention to their own health. As the country is committed to improving the medical insurance system, the consumption utility of urban residents will be improved, and residents will enjoy a large number of preferential subsidies such as medical insurance, etc. Consumers will gradually increase their willingness to consume, and consumer satisfaction will increase significantly.

Combined with the third section and this section, based on the above research work, we come to the following conclusions: First, the consumption level of urban residents in China is increasing year by year. From 1993 to 2016, the per capita annual consumption expenditure of urban residents and the total retail sales of consumer goods in China increased steadily and substantially, and the per capita consumption level increased continuously, and the growth rate of per capita consumption has been increasing. Second, from 1993 to 2016, the optimization speed of China's consumption structure maintained a rapid development momentum, predicting that China's consumption structure will continue to be optimized in the future. Traffic consumption and communication consumption have the greatest impact on the optimization of consumption structure, followed by food, education, etc. These types of consumption are high-quality consumption. The increase in such consumption has undoubtedly intensified the optimi- 
zation and upgrading of China's urban consumption structure. Third, consumer satisfaction is constantly improving. Emerging consumption and potential consumption have been rising in urban residents' consumption. These two types of consumption have attractive attributes and unit attributes. Increasing emerging consumption and potential consumption can bring huge satisfaction to consumers, leading to significant improvement of consumer satisfaction in recent years.

\section{Policy Recommendations}

Consumption has gradually increased its contribution to economic growth and become an important force driving economic growth, while supply-side reform is the internal driving force of consumption upgrading. The supply-side reform is mainly through the allocation of labor, land, capital, institutional creation, innovation and other factors to vigorously develop the real economy. Zhang Ting (2019) believes that China should further reduce the proportion of the structure of the primary industry, increase the proportion of the secondary industry structure and the proportion of the tertiary industry structure to meet the consumer demand [20]. Therefore, based on the background of supply-side reform and the actual development of China, this paper proposes the following specific measures to promote consumption upgrading:

1) Improve the quality of labor supply and the quality of employment, and increase the income of residents. The income level directly determines the consumption power of residents and is the most important factor affecting the consumption upgrade. At present, the income of most residents comes from wage income, but the ratio of wage income to GDP is gradually decreasing, and frictional unemployment and structural unemployment rate are getting higher and higher. The government should appropriately improve the pattern of income distribution among residents and achieve fair distribution. On the other hand, the government improves the labor market mechanism to cultivate and improve the professional skills of labor force according to different needs of various enterprises, so that workers can better adapt to the needs of economic development and industrial structure optimization and upgrading, reduce frictional unemployment and structural unemployment, and promote China's consumption upgrading.

2) Improve the quality of transportation and communication products and drive innovation to lead development. The demand for transportation and communication is second only to food and housing. The national policy should vigorously support the research and development of local enterprises in China's transportation and communication industry, adhere to independent innovation, and deploy the innovation chain around the industrial chain with the increasing consumer demand as the guide to develop high-quality and low-cost transportation and communication products. Let more people of different consumption levels enjoy high-quality products and services, and promote the overall con- 
sumption upgrade. In addition, Adam Smith believes that "the growth of productivity and wealth is caused by the division of labor. The degree of division of labor is restricted by the size of exchange capacity, that is, the scope of the market. The size of the market is determined by transportation conditions." The transportation and communication industry can bring external economic effects. The construction of traffic in remote areas should be improved, and the development of the commerce and trade circulation industry [21] should be used to drive the improvement of its overall income level to promote the wider consumption upgrade in China.

3) Reducing the transaction costs of residents' purchases and speeding up the reform of the housing system. Housing is also a hot spot for residents to consume. People often improve the quality of life by improving the living environment. Therefore, the overall idea of deepening the reform of the housing system should be to ensure the sound development of the housing market and build a new housing system. The state should increase the transaction costs of speculative housing, curb the phenomenon of "real estate speculation", and clarify that "the house is used for living rather than for speculation". The government can increase fiscal revenue by charging the standard price for commercial land use, optimize the tax system in the transaction, reduce transaction costs, maximize the interests of consumers, and enable residents to have more in transportation, medical care, etc. The budget, thus is promoting the upgrading of our consumption.

4) Improve the medical security system and develop scientific and technological medical devices. China needs to deepen the reform of the medical and health care system, strengthen the supervision of medical services, and establish a national health-service system that can meet diversified needs. On the other hand, the government must increase investment in the development of science and technology. China has basically achieved the independent production of conventional medium and low-end medical equipment, but the medical equipment market is small in scale, low in industrial concentration, and low in equipment level in primary medical institutions. As for the development of high-end medical equipment, most of the core technologies are in the hands of foreign companies, it is difficult for Chinese enterprises to form independent innovation brands. Therefore, China must grasp the market demand orientation, introduce foreign advanced technology, use the advantages of localization to expand the product line of high value-added products, foster the development of independent innovation enterprises, and jointly promote the consumption upgrade of China through the sound medical security system and the development of high-tech medical devices.

\section{Conclusion}

In the pattern of economic growth, domestic demand is the key, and consumption in domestic demand is the core factor. The report of the $19^{\text {th }}$ National Congress of the Communist Party of China clearly pointed out that "we must further 
improve the system and mechanism for promoting consumption and further enhance the fundamental role of consumption in economic development". Therefore, the transformation of China's economic structure should accelerate the transition from investment and export orientation to consumption orientation. According to the National Bureau of Statistics, from 1997 to 2018, the contribution rate of final consumption expenditure to GDP has steadily increased. With the rapid increase in consumption and the rapid growth of the consumer market, domestic consumption has played a huge role in promoting economic growth. Consumption upgrading has become the first driving force for economic growth and an important "stabilizer" for driving smooth economic operations. Therefore, "actively comply with and grasp the general trend of consumption upgrading, continue to promote consumption, improve consumption level, expand consumption scale, and enhance the rationality of consumption structure is an important measure to do a good job in the current and future consumption promotion work" has a very important theoretical guidance and practical significance.

\section{Conflicts of Interest}

The author declares no conflicts of interest regarding the publication of this paper.

\section{References}

[1] Mao, Z.G. and Xie, C. (2019) Xi Jinping's Important Exposition on Consumer Economy-Reality Basis, Theoretical Basis and Main Content. Consumer Economy, $35,3-11$.

[2] Sun, H., Mao, Z.G. and Gui, H.Q. (2019) China's Residents' Consumption Inequality: Examination and Response. Modern Economics Discussion, No. 4, 8-14.

[3] The State Council of China (2019) The Government Work Task of the State Council's Government Work Report in 2019 (Summary). Construction Machinery Technology and Management, 32, 1.

[4] Xi, J.P. (2018) Decisive Victory to Build a Well-Off Society in an All-Round Way to Win a Great Victory in Socialism with Chinese Characteristics in the New Era-Report at the 19th National Congress of the Communist Party of China (Excerpt). Heihe Academic Journal, No. 1, 2+193.

[5] Li, P.M. (2018) The Overall Situation of Residents' Consumption Development. China Economic and Trade Guide, No. 12, 35-36.

[6] Shi, X.R. (2019) Research on Consumption Upgrading Phenomenon and Future Development Trend. Knowledge Economy, No. 7, 60-61.

[7] Ma, Z.G. (2018) Does the Rise of Pin Duo Duo Mean a Demotion of Consumption? Internet Economy, No. Z2, 86.

[8] Ma, Q.Z. (2018) Re-Recognizing Consumption Upgrade and Consumption Adjustment. Xiaokang, No. 24, 54-55.

[9] Xue, J.M. and Yan, M. (2019) Consumption Upgrading or Consumption Downgrade: Estimation of Rural Residents' Data from Shanghai-Also on the Effectiveness of Engel's Coefficient in Measuring Residents' Living Standards. Shanghai Econo$m y$, No. 2, 56-67. 
[10] Huang, J. and Li, J.K. (2018) Characteristics, Measurement and Development of China's Consumption Upgrade. China's Circulation Economy, 32, 94-101.

[11] Li, M. (2018) Consumption Upgrade or Consumption Downgrade. Guangguang, No. 9,11 .

[12] Kuang, X.M. (2018) The Judgment of Consumption Downgrade Is Not Established. Shandong Economic Strategy Research, No. 7, 4.

[13] Tan, H.J. (2018) Who Is in Charge of the Promotion and Downgrade of Consumption? China Business Times, 2018-09-25 (003).

[14] Sun, F. (2019) Some Understandings about the "Upgrade" and "Downgrade" of Consumption. People's BBS, the Academic Front, No. 2, 13-19.

[15] Shi, M.M., Jiang, Z. and Zhou, X.Y. (2019) Consumption Upgrade or Consumption Degradation. China Industrial Economy, No. 7, 42-60.

[16] Hou, X.S. (2019) Giving Full Play to the Basic Role of Consumption to Promote the Smooth Operation of the Economy-A Review of the "Foreign Symposium on Consumer Economics Research" in 2019. Consumer Economy, 35, 93-96.

[17] Sun, X.J., Lu, W. and Zhang, W. (2019) Consumption Downgrade or Consumption Stratification?-Research on Dynamic Characteristics of China's Household Consumption Trends. Commercial Research, No. 8, 25-35.

[18] Wen, Q.X. and Ran, J.F. (2005) The Harmony of Consumption Structure and Industrial Structure: Harmony and Its Measurement. China Industrial Economy, No. $8,14-19+104$.

[19] Wan, L.L., Gan, J.F. and Yu, X.Y. (2010) Time-Based Global Principal Component Analysis Method Based on Matlab and Its Application. East China Economic Management, 24, 150-153.

[20] Zhang, T. (2019) China's Industrial Structure Adjustment Trend under the Background of Consumption Structure Upgrading. Business Economics Research, No. 3, 175-177.

[21] Yang, J. and Luo, Y.M. (2018) The Impact of the Development of Commerce and Trade Circulation Industry on the Consumption Structure of Urban and Rural Residents-Based on the Perspective of Consumption Upgrade. Research on Business Economy, No. 24, 41-44. 